\title{
Disfrutar la vida o disfrutar de la vida. ¿La alternancia entre régimen directo y preposicional es un fenómeno de diátesis?
}

Enjoy life or enjoy from life. Is the alternation between direct regime and prepositional regime a diathesis phenomenon?

Blanca Elena Sanz Martin

Cristina Eslava Heredia

\section{Resumen}

El presente trabajo analiza los verbos disfrutar, necesitar y cuidar, que muestran una alternancia entre el régimen directo y preposicional. Son distintos semánticamente, pero dado que el fenómeno se da en un grupo tan pequeño de verbos, se busca explorarlos para establecer algún factor transversal a todo el grupo. En principio, se pretende establecer los factores que condicionan la alternancia. Además, se someten los datos a los parámetros de la diátesis antipasiva, tanto desde el análisis cualitativo de los contextos en corpus, como desde una perspectiva cuantitativa.

Palabras clave: diátesis antipasiva, alternancia de régimen, individuación, completud del evento, aspecto

\begin{abstract}
The present work analyzes the verbs disfrutar, necesitar and cuidar, which show an alternation between the direct and the prepositional regimen. These verbs are semantically different, but given that the phenomenon belongs to a very small group of verbs, the aim of this work is to analyze them in order to discover transversal factors for all the group. The initial purpose is to establish the factors that conditions the alternation. Also, data are analiyzed with parameters of the antipassive diathesis. These data derive from qualitative and quantitative analysis of the contexts appearing in the corpus.
\end{abstract}

Key words: antipassive diathesis, alternation of regimen, individuation, event completion, aspect

Blanca Elena Sanz Martin, Universidad Autónoma de Aguascalientes (México)blancasanz27@hotmail.com Cristina Eslava Heredia, Universidad Autónoma de Aguascalientes (México)ristina_eslava@hotmail.com

Recibido: julio 2016 / Aceptado: octubre 2016

DOI 10.17710/tep. 2017.3.1.2sanzmartineheredia 


\section{Introducción}

En español existen algunos verbos que alternan la posibilidad de construirse con un complemento directo (CD) o un complemento preposicional (CP), como se muestra en los siguientes ejemplos tomados de Alarcos (1978, p. 11):

(1) a. Trató la pulmonía con penicilina/ trató de la pulmonía con erudición.

b. Pensad ese asunto/pensad en ese asunto.

c. El chico entiende los negocios/el chico entiende de negocios.

En algunos casos, la alternancia de complemento representa un cambio de significado léxico, lo cual indica que se trata de verbos distintos, como ocurre en (1a). No obstante, en otros casos, los verbos pueden complementarse mediante una frase nominal (CD) o una frase prepositiva (CP) sin que cambie su significado léxico, como ocurre en los ejemplos (1b) y (1c). De hecho, ciertos verbos permiten que el mismo referente se codifique sintácticamente como $\mathrm{CD}$ o $\mathrm{CP}$, como se ilustra en los siguientes ejemplos:

(2) a. Juan disfruta la noche.

b. Juan disfrutade la noche.

(3) a. Juan necesita una sirvienta.

b. Juan necesitade una sirvienta.

(4) a. Juan cuida su negocio.

b. Juan cuidade su negocio.

Obsérvese que los argumentos de las oraciones presentan el mismo contenido referencial. La diferencia estriba en la manera en que se codifica sintácticamente el segundo argumento. El presente trabajo analiza los verbos del tipo ilustrado en (2), (3) y (4), es decir, aquellos que presentan una alternancia entre el régimen directo y preposicional sin que exista un cambio del significado léxico.

Este objeto de estudio implica una dificultad teórica, pues si consideramos el principio funcional de que dos manifestaciones formales distintas suponen dos significados diferentes, habría que cuestionarse en qué consiste la diferencia semántica entre ambos tipos de régimen. Asimismo, los verbos que alternan entre la rección directa y la preposicional suponen también el problema de una alternancia en la transitividad oracional. 
En los pares ilustrados en (2), (3) y (4), cada oración describe la misma realidad objetiva; no obstante, en (2a), (3a) y (4a), las oraciones son transitivas y en (2b), (3b) y (4b), intransitivas. Aunque tanto el CD como el CP son exigidos por el verbo desde el punto de vista semántico, desde el punto de vista sintáctico el CD es un elemento central, mientras que el CP es un elemento oblicuo (cf. Cooreman, 1994, pp. 65-66).

Entre la bibliografía afín al tema, encontramos que Cifuentes (2010, p. 30) identifica el fenómeno de alternancia entre régimen directo y preposicional, pero no llega a explicar las diferencias semánticas que podrían subyacer de estas, pues el trabajo se enfoca en el aspecto sintáctico, ya que su objetivo consiste en mostrar cómo un mismo verbo puede ser asociado con un número diferente de argumentos en distintos usos, a la vez que puede haber cambios en las realizaciones gramaticales de los argumentos.

Asimismo, Jiménez Norberto (2009) identifica el fenómeno y describe el estatus argumental de la frase prepositiva en términos sintácticos. Si bien la autora reconoce que la alternancia implica ligeros cambios semánticos, el objetivo del trabajo no es explicar dichos matices de significado en las estructuras estudiadas.

Cano Aguilar (1999), respecto a estos verbos y a tenor del concepto de 'régimen', señala que:

la presencia de la preposición depende sólo del verbo en cuestión, del que llega a considerarse una expansión meramente formal, hasta el punto que puede desaparecer dejando una construcción meramente transitiva (disfruta (de) la vida). Se cumplen, pues, las condiciones exigidas para hablar del 'régimen' tanto como exigencia sintáctico-semántica cuanto como exigencia formal. (p. 1815).

Según observa Cano Aguilar (1999): "Parece haber alternancia libre (al menos no se han establecido condicionantes claros de uso) entre objeto directo y complemento con de en verbos de 'actuación' como cuidar o usar, o de sensación como disfrutar o gozar' (p. 1824).

En un primer momento, nuestro análisis partió de la hipótesis de que la alternancia verbal entre la rección directa y la preposicional se podría explicar en términos de un fenómeno de diátesis antipasiva, en tanto que el $\mathrm{CD}$, que es un elemento central, se transforma en oblicuo. Esta hipótesis explicaría la presencia de la preposición como un elemento que icónicamente formaliza la degradación de un actante central en la construcción (cf. Givón, 1984, p. 164). 
Un trabajo previo que estudia el fenómeno desde la perspectiva de la hipótesis planteada es el realizado por Bogard (1999), quien aborda la alternancia en términos de un fenómeno de diátesis antipasiva. Este autor ofrece un punto de partida al estudio de estructuras alternantes entre régimen directo y preposicional y explica tal alternancia en términos de un fenómeno de voz antipasiva. Sin embargo, el objeto de estudio del trabajo se centra en las alternancias que implican la presencia del clítico pronominal se como un clausurador argumental. Véanse los siguientes ejemplos del autor (Bogard 1999, p. 305):

a. Juan aprovecha tu experiencia.

b. Juan se aprovecha de tu experiencia.

(6) a. Juan compadece a los muchachos.

b. Juan se compadece de los muchachos.

De acuerdo con el autor, la destransitivización supone un cambio de significado, en el cual el agente adquiere un grado de afectación que en su correlato transitivo no presenta. Esta afectación se traduce en el grado de interés o responsabilidad que el primer argumento tiene sobre el segundo, mientras que el elemento oblicuo toma la interpretación de un punto de referencia, al cual el autor tipifica como procedencia secuencial retrospectiva (Bogard 1999, pp. $322,326){ }^{1}$

Por lo anterior, una vía de análisis de la alternancia de régimen directo y régimen preposicional de los verbos necesitar, cuidar y disfrutar partiría de la hipótesis de que tal alternancia podría obedecer a un cambio de diátesis antipasiva, con lo cual se explicaría por qué un elemento central como el objeto directo puede manifestarse sintácticamente como un elemento oblicuo ante la pérdida de prominencia semántica. Así, el objetivo de este trabajo es estudiar la alternancia de régimen a la luz de los parámetros de la antipasiva para validar o no la hipótesis planteada.

Maldonado (2004) rechaza la propuesta de Bogard (1999) del clítico se como marca de voz antipasiva, pues aduce que en la lengua española no se cumplen a cabalidad las funciones básicas de esta voz. Para este autor, no solo se trata de convertir al OD en oblicuo,

\footnotetext{
${ }^{1}$ Este concepto lo retoma de Rojas Nieto (1988, p. 73). Sirve para marcar un punto de referencia con respecto al cual el agente lleva a cabo el evento denotado por el verbo y con respecto al cual se justifica el valor activo de lo expresado en él. En este caso, según Bogard, el referente introducido por la preposición es concebido como necesariamente anterior o preexistente al agente y a la acción.
} 
sino que el objeto adquiera en su constitución una menor prominencia. Para entender mejor el problema de la propuesta de Bogard habrá que entender la función de la voz antipasiva.

\title{
2. La diátesis antipasiva
}

Como hemos mencionado, este trabajo partió de la hipótesis de que el fenómeno de la alternancia se puede explicar en términos de una diátesis antipasiva. Por lo que en esta sección trataremos brevemente dicho concepto.

La categoría gramatical de diátesis se vincula con la categoría de voz, que se refiere a una categoría gramatical que expresa las relaciones entre predicado y sus argumentos o actantes (García Miguel, 1995, p. 107), la cual también puede ser entendida como un cambio del esquema sintáctico relacionado con el número de elementos centrales de la oración (Moreno Cabrera, 1984).

Tradicionalmente, el término voz, suele emplearse como equivalente a diatesis. No obstante, los cambios de esquema sintáctico (por ejemplo: causatividad, anticausatividad, construcciones aplicativas, etc.) implican cambios de diátesis, pero no necesariamente cambios de voz (cf. Moreno Cabrera, 1984, pp. 25-26),

\begin{abstract}
puesto que no necesariamente dan lugar a una categoría gramatical de predicado, con formas de expresión diferenciadas. La vOz permite cambiar la(s) diátesis básica(s) en diátesis marcada o derivada. Es decir, los términos marcados en la categoría de voz indican una correspondencia entre funciones sintácticas y argumentos distinta de la esperada con cierto predicado (García Miguel, 1995, pp. 108).
\end{abstract}

Típicamente, los fenómenos de voz que hacen posible la disminución de un elemento central son la pasiva y la antipasiva, en tanto que sirven ambas como mecanismos de destransitivización (Givón, 1984, pp. 164). Aunque el fenómeno de la antipasiva es propio de las lenguas ergativas, también ha sido aplicado a ciertas construcciones de las lenguas nominativo-acusativas (Cooreman, 1994, pp. 65-66; García Miguel, 1995, p. 113).

Como veremos a continuación, nuestras construcciones tienen en común con las antipasivas el hecho de que el objeto directo se degrada, es decir, pasa de ser un elemento más central a uno más periférico. No hay una marca formal en el verbo, pero sí hay un cambio en el esquema sintáctico que permite la disminución de los elementos centrales:

$$
\mathrm{S}+\mathrm{V}+\mathrm{FN}(\mathrm{CD})>\mathrm{S}+\mathrm{V}+\mathrm{FP}(\mathrm{CP})
$$


Cooreman (1994, p. 49) propone la existencia de dos tipos de antipasivas. La primera se define en términos semánticos y pragmáticos y la segunda en términos estructurales. La antipasiva del primer tipo, es decir, empleada por razones semántico-pragmáticas, se utiliza para indicar cierto grado de dificultad de la fuerza de la actividad de A en un O identificable (Cooreman, 1994, p. 51). A primera vista, nuestras construcciones podrían pertenecer al primer tipo, puesto que no hay marca formal en el verbo, pero sí parecieran cumplir una función semántica y pragmática.

Recordemos que en la voz pasiva de una lengua nominativa-acusativa se promociona al participante $\mathrm{P}$ a la posición de Sujeto; mientras que se degrada al participante A, que ocupa la función original del Sujeto, mediante una forma oblicua, que fungirá como complemento Agente (C. Ag). Como resultado, la pasiva presenta un participante menos que la activa correspondiente, es decir, el actante A deja de ser participante central, tal como se ilustra en el Cuadro 1:

Cuadro 1. Activa y pasiva

\begin{tabular}{|l|lc|ll|}
\hline & \multicolumn{2}{|l|}{ Activa/directa } & Pasiva \\
\hline & S (A) & O (P) & S (P) & C. Ag (A) \\
\hline Transitiva & Nom & Acus & NomObl & \\
\hline Intransitiva & Nom & & Nom \\
\hline
\end{tabular}

El equivalente funcional de la pasiva en las lenguas ergativas es la construcción antipasiva, en donde $\mathrm{A}$ y $\mathrm{O}$ en una construcción activa ergativa se marca como ergativo y absolutivo, respectivamente; en una antipasiva, A se marca como una $\mathrm{FN}$ absolutiva, y O (si es que aparece), como un oblicuo, tal como lo ilustra Maldonado (2004, p. 119) con el siguiente cuadro adaptado que reproducimos aquí:

Cuadro 2. Activa y antipasiva

\begin{tabular}{|l|ll|l|}
\hline & \multicolumn{2}{|l|}{ Activa/directa } & Antipasiva \\
\hline & S (A) & O (P) & S \\
\hline Transitiva & Erg & Abs & AbsObl \\
\hline Intransitiva & Abs & Abs \\
\hline
\end{tabular}

Para Maldonado y otros autores mencionados en su artículo, este comportamiento 
general presenta manifestaciones específicas con impacto comunicativo, entre las cuales menciona una serie de operaciones de reducción de transitividad como consecuencia de procesos de democión del objeto absolutivo (Maldonado, 2004, p. 119-120):

1. La antipasiva hace que la actividad no sea totalmente realizada debido a que la marcación de oblicuo genera cierto grado de dificultad para que el agente produzca el efecto deseado en el objeto.

2. Aunque haya contacto con el objeto, en la construcción antipasiva el evento es incompleto.

3. En lenguas como el samoano, el contacto se puede dar, pero ahora la construcción designa un significado partitivo.

4. En casos quizá más sutiles la construcción reduce la transitividad de la cláusula al bajar el grado de individuación/identificación del objeto.

Así, el autor menciona que "la función central de la construcción antipasiva es imponer algún tipo de distancia real o conceptual entre el agente y el paciente de manera que el último no está en disposición del sujeto para ser afectado" (Maldonado 2004: 121). Por lo anterior, el perfil se mantiene en el agente, mientras el objeto y su afectación quedan relegados a un segundo plano.

Maldonado recuerda que existen correlatos aspectuales que no se deben dejar de considerar, como, por ejemplo, "que mientras las pasivas tienden a manifestarse en aspecto perfectivo, las antipasivas tienden a hacerlo en formas aspectuales de corte imperfectivo" y esto tiene efectos en varias dimensiones (Tsunoda 1988, citado enMaldonado, 2004, pp. $122-$ 123) de modo que:

1. La marcación imperfectiva sirve para designar eventos habituales que comúnmente se asocian con designaciones de corte genérico.

2. Si bien no siempre el evento es habitual sí puede ser repetitivo.

3. El evento imperfectivo tiende a no ser puntual. Mientras que la activa directa permite que haya un evento ubicado en un espacio temporal, la antipasiva sirve para designar la potencial ocurrencia de un evento en un tiempo cualquiera.

Por tanto, en las construcciones antipasivas hay un énfasis en "eventos incompletos generalmente no ubicados en tiempo y espacio alguno y en los que no hay afectación completa del objeto dado su bajo nivel de individuación". En resumen, según Maldonado 
(2004, p. 123), las funciones básicas de la antipasiva se pueden esquematizar con los siguientes parámetros:

Cuadro 3. Parámetros de antipasiva (Maldonado, 2004: 123)

\begin{tabular}{|l|l|l|}
\hline EVENTO & BAJO NIVEL DE & ASPECTO \\
INCOMPLETO & INDIVIDUACIÓN & IMPERFECTIVO \\
\hline Baja afectación & Objeto indefinido & Habitual \\
Falta de contacto & Objeto no especifico & Repetitivo \\
$\begin{array}{l}\text { Evento incompleto } \\
\text { Significado partitivo }\end{array}$ & Objeto genérico & No puntual \\
\hline
\end{tabular}

A partir de estos parámetros, el autor hace una revisión de los ejemplos de las construcciones estudiadas por Bogard (1999, citado enMaldonado 2004, p. 123) como antipasivas, los cuales reproducimos aquí:

(7) a. Juan se aprovechóde tu experiencia

b. Juan se compadeció de los muchachos

c. Juan se despidió de los excursionistas

d. El gobierno no se desviará de la política económica actual

e. Carlos se evadió de la justicia

f. Juan se lamentó de tus palabras

g. Juan se soltó de la cuerda

Las observaciones de Maldonado (2004, pp. 123-124) son las siguientes: en los ejemplos "sobresale el hecho de que los complementos oblicuos son de alta individuación", "los valores aspectuales son notoriamente perfectivos" y "ocurren todos en pretérito y en ningún caso designan acciones habituales, iterativas o genéricas. Se trata de acciones claramente ancladas en un momento puntual en relación con entidades específicas y bien determinadas". El autor reconoce que es "indudable que en todos estos ejemplos se da un fenómeno de baja transitividad. Pero igualmente nítido es que tal fenómeno no parece obedecer a las propiedades de la voz antipasiva".

Maldonado considera la propuesta de Bogard como: "la imposición del patrón de las lenguas ergativas sobre una lengua radicalmente distinta" (p. 124). El problema para 
Maldonado radica en que "borra la distinción entre una serie de construcciones que en esta lengua contrastan con claridad. Aprovecharse de alguien difiere notablemente de montarse en la mula o de soltarse de la cuerda y éstas contrastan de manera verdaderamente radical de jactarse y de arrepentirse de algo" (p. 124).

Estos parámetros retomados de la bibliografía especializada por Maldonado también los retomaremos más adelante para verificar si nuestras construcciones pueden considerarse o no antipasivas.

\section{Metodología y corpus}

El grupo de verbos que alternan rección directa y preposicional es muy pequeño y heterogéneo desde la perspectiva semántica. Para este estudio elegimos los verbos disfrutar (que se agrupa con el verbo gozar), necesitar (que se agrupa con los verbos requerir, precisar y, en usos dialectales, usar y ocupar) y el verbo cuidar.

Presentamos la clasificación semántico-sintáctica de nuestros tres verbos de acuerdo con la clasificación ofrecida por la Base de Datos de Verbos, Alternancias de Diátesis y Esquemas Sintáctico-Semánticos del Español (ADESSE). El verbo disfrutar se clasifica como un verbo de sensación + uso que selecciona un experimentador y un estímulo. Su significado se refiere a 'sentir alegría o placer con la adquisición o uso de algo' (gozar). Necesitar es un verbo de posesión que requiere un poseedor y un poseído y se refiere a 'tener necesidad de algo o alguien o exigirlo’. Por último, cuidar se clasifica como un verbo de percepción o disposición. Como percepción, se requiere un perceptor y un percibido y significa 'dedicar [alguien] atención e interés a [algo o a alguien] para que permanezca en buenas condiciones' (dedicar), mientras que la acepción de disposición implica un actor y una acción y significa 'cuidar [alguien] [de hacer o evitar algo]'. El significado de cuidar como percepción, según la información mostrada en la base de datos, resulta dudosa ${ }^{2}$. De acuerdo con los datos obtenidos de nuestro corpus, los límites entre una acepción y otra no son discretos, pues el evitar la realización de una acción implica la percepción constante de la entidad cuidada.

Los tres verbos que seleccionamos, disfrutar, necesitar y cuidar, son distintos semánticamente, pero dado que el fenómeno de alternancia se da en un grupo tan pequeño de verbos, hemos querido explorarlos para establecer, de ser posible, algún factor transversal a todo el grupo. El único factor que justifica la elección de estos verbos es de índole

\footnotetext{
${ }^{2}$ Tal acepción aparece con un signo de interrogación. 
sintáctico: la alternancia de régimen directo y el régimen preposicional; es decir, no se tomó en cuenta el significado léxico de éstos.

Los datos para este estudio fueron obtenidos de El Corpus del Español de Mark Davies. Se extrajeron datos de manera aleatoria del siglo XX de todas las variedades dialectales ${ }^{3}$. Se seleccionaron solo formas conjugadas con complementos conformados por una $\mathrm{FN}$ con o sin preposición. No se tomaron en cuenta para este estudio complementos oracionales subordinados ni formas en infinitivo.

\section{Resultados}

En una primera etapa analizamos los datos recolectados de El Corpus del Español de Mark Davies para establecer los factores que condicionan la alternancia dentro de los elementos que integran las construcciones. La siguiente tabla resume la distribución de la alternancia entre régimen directo y el prepositivo de los tres verbos seleccionados.

Tabla 1. Frecuencia de uso del $C D$ vs. $C P$

\begin{tabular}{|c|c|c|c|}
\hline Verbo & CD & CP & TOTAL \\
\hline Necesitar & $80 \%$ & $20 \%$ & $100 \%(419)$ \\
& $(334 / 419)$ & $(85 / 419)$ & \\
\hline Cuidar & $73 \%$ & $27 \%$ & $100 \%(183)$ \\
& $(134 / 183)$ & $(49 / 183)$ & \\
\hline Disfrutar & $15 \%$ & $85 \%$ & $100 \%(345)$ \\
& $(50 / 345)$ & $(295 / 345)$ & \\
\hline
\end{tabular}

Podemos observar que los tres verbos en menor o mayor medida muestran esta alternancia entre el régimen directo y el preposicional. Los verbos transitivos necesitar y cuidar mostraron una mayor preferencia por la rección directa, $80 \%$ y $73 \%$ respectivamente. Por otra parte, disfrutar es un verbo que tiende al régimen preposicional con una frecuencia del 85\% de los casos, lo que coincide con lo señalado por Cano Aguilar (1999, pp. 1824-1825).

\subsection{Los parámetros de la antipasiva}

Al someter los datos a los parámetros de la antipasiva, observamos que no se cumple íntegramente ninguno de ellos, como se expondrá a continuación.

\footnotetext{
${ }^{3}$ Se tomó aproximadamente un $10 \%$ del total de ocurrencias arrojadas en cada uno de los verbos. 


\subsubsection{Evento incompleto}

En los ejemplos de (8), con el verbo necesitar, contrastamos los datos según el parámetro de "evento incompleto". Obsérvese que se trata de construcciones negativas que indican falta de necesidad de algo; por tanto, lo esperable sería la presencia de CP, pues no se lleva a cabo el evento o estado de cosas que predica el verbo.

(8) a. No necesitaba de imaginaciones ahora misia (sic) Marisabel para sentirse atribulada [Chile: Barrios, Eduardo, Gran señory rajadiablos, 1981]

b. Yo no necesitaba de excusas. Sabía muy bien lo que ocurrió con Nicolás. [Riquelme, Yula, Puerta, 1980]

c. Segundo tanto de Laferrere a Brown y explosión: aunque Lanús no necesitara de ese gol en otro estadio para coronarse, fue como un bálsamo [Argentina: Diario La Prensa, mayo de 1992]

Lo esperable, entonces, es que las construcciones afirmativas que expresan la existencia de necesidad y, por tanto, la realización de la predicación, seleccionen un régimen directo; sin embargo, las construcciones en afirmativo también aparecen acompañados por un CP, como en (9), o incluso con verbos en tiempo perfectivo, como en (10):

(9) Ella desde luego puede recomendar a cualquiera si quiere pero sabe lo que le conviene, aquí necesitamos de confianza [Paraguay: Rodríguez, Guido, Cuentos, 1993]

(10) a. Esa vida y esa masacre no duraron horas, ni días, sino años, se necesitó de años para matar tantos millones de seres [Paraguay: Gertopan, Susana, Barrio Palestina, 1988]

b. En Noruega, el fascismo atrajo a algunos simpatizantes notables como Vidkun Quisling y el premio Nobel de Literatura KnutHamsun, pero del mismo modo necesitó de la ocupación alemana para disfrutar de algún poder político [Encarta, Enc. Fascismo]

Ahora bien, con el verbo disfrutar aparentemente el parámetro de "evento incompleto" parece funcionar. Contrástese los ejemplos de (11) y (12). El ejemplo de (11) contiene el adverbio poco, con el cual se quiere expresar que el evento no es alcanzado del 
todo o es parcialmente alcanzado, de ahí que lo esperable es la presencia de CP; mientras que los ejemplos de (12) contiene la frase al máximo que supondría el alcance del evento y, por tanto, la presencia de un $\mathrm{CD}$.

(11) Mrs. Todman se pasó el mediodía con los lentes en la mano dirigiendo a los mozos. Vestida en grises - azules y el cabello negro alaciado, prefirió hablar en inglés y disfrutó poco de la reunión. [Arg: Cronista:637_4DE].

(12) a. Me dijo: - lo que suceda será lo debido; disfrutemos al máximo el sabor de esta empresa [Argentina: Mourelle, Daniel, Miramar, 1999]

b. Sus triunfos son el mejor aliciente para seguir adelante en un deporte que lo hace feliz y que disfruta al máximo: el atletismo [Costa Rica: Diario, La nación, 1998]

A primera vista, el parámetro de la completud del evento parece funcionar. No obstante, una vez más encontramos datos que expresan eventos alcanzados cuyo complemento se formaliza como CP, sin pérdida de gramaticalidad, tal como se observa en los ejemplos de (13), donde los adverbios mucho e intensamente nos permiten interpretar la acción de disfrutar o el estado de disfrute como algo que se ha alcanzado ya, es decir, como un evento completo; no obstante, el complemento verbal se codifica como preposicional.

a. El disfruta mucho del estudio, de la mesa de grabación que para mí no es un instrumento [México: Diario de Yucatán, 1971]

b. Inf.a. - Bueno, no tiene nada de raro que un muchacho como nuestro hijo, de doce años más o menos, disfrute intensamente de... de su primer viaje solo [Chile: Habla culta, 1941]

Como vemos, el parámetro de la completud del evento no constituye un factor que determine la alternancia entre el régimen directo y el prepositivo.

\subsubsection{Nivel de individuación}

Las construcciones antipasivas suponen que los complementos poseen un bajo grado de individuación y, por tanto, es esperable que aparezcan introducidos por una marca morfológica o prepositiva; mientras que las estructuras activas preferirían un régimen directo 
con objetos con un alto grado de individuación. Contrástese los ejemplos (14) y (15) a continuación. Los objetos prepositivos de (14) presentan una baja individuación, en tanto que sus referentes no son específicos; en cambio, el objeto directo de (15) muestra una alta individuación. Entendemos como especificidad el grado de reconocimiento de las entidades por parte del oyente.

(14) a. Me imagino la reacción de sorpresa que habrán causado estas informaciones, cuando menos apresuradas, entre los doctores [...] que, día a día, cuidan en sus respectivos hospitales de la salud de los seropositivos y asintomáticos. (no específico) [España: ABC, 1979]

b. Los machos mueren después del apareamiento y las trabajadoras recogen alimentos, cuidan de los jóvenes [hormigas] y defienden la colonia [Encarta: Enc. Hormigas]

c. Admiraban las preocupaciones de Ángel, cómo cuidaba en lo posible a su mujer [Chile: Orrego, Luis, Casa grande: escenas de la vida, 1908]

En los ejemplos de (14) las frases la salud de los seropositivos y asintomáticos y los jóvenes en el contexto en que aparecen no son entidades específicas, por tanto, es esperable que aparezcan introducidos por la preposición. Por el contrario, se esperaría que estructuras activas prefieran un régimen directo con objetos con un alto grado de individuación, como en el ejemplo (15), pues el referente del CD a su mujer es específico, esto es, identificable para el oyente.

A pesar de lo anterior, lamentablemente, el parámetro de individuación tampoco se cumple sistemáticamente, pues es posible encontrar también datos que presentan objetos altamente individuados introducidos con la preposición de, como el ejemplo de (16).

(16) ¿La has tenido alguna vez? — sí, pero murió... Cirrosis hepática. —oh. ¿Bebía mucho? —mucho. —y ahora, ¿quién cuida de ti? —Peter y los chicos [México: Fernández, Xavier, Kensington Gardens, 2001]

Del mismo modo, es posible encontrar objetos con baja individuación con régimen directo, tal como lo testimonia el ejemplo de (17), donde la frase enfermos de Ébola o fiebre de Lassa no es introducida por la preposición de,como sería lo esperable. 
El personal sanitario que cuida a enfermos de Ébola o fiebre de Lassa está expuesto a un riesgo elevado de infección [Encarta: Enc: Fiebre hemorrágica]

Por tanto, el parámetro de baja individuación para explicar la presencia de la preposición de como formalización de la función de antipasiva no tiene incidencia como factor de alternancia entre el régimen directo y el preposicional.

\subsubsection{Aspecto imperfectivo}

El último parámetro que revisamos está relacionado con la dimensión aspectual. Las construcciones antipasivas, según la bibliografía conocida, tienden a presentarse en construcciones imperfectivas, ya sea porque el evento es habitual, repetitivo o no puntual.

Contrástese los ejemplos de (18) y (19) con el verbo cuidar. En ambos ejemplos, el verbo está en tiempo presente y el modo aspectual se interpreta como una acción habitual o actividad. No obstante, mientras que en (18) el complemento es de tipo preposicional, en (19) es directo.

En el hospital de campaña para heridos de guerra de Augsburgo uno de ellos presta servicios para la salud corporal de los soldados, y el otro cuida de su salud espiritual [Perú: Garcí, Raquel (traductora), “Adam Birner”, 1998] Si una empresa cuida a sus trabajadores, a ésa (sic) empresa se le premia y va a pagar menos cuota [ México: Diario La República, 1987]

En el caso de los verbos en tiempos que nos remiten a modos aspectuales perfectivos como una actividad puntual, encontramos que puede haber una elección por el régimen directo, tal como lo atestigua el ejemplo de (20); pero también encontramos eventos puntuales con régimen prepositivo, como ocurre en los ejemplos de (21).

(20) fui acompañando tu crecimiento con mis manos, adivinando tus diferentes tamaños desde que cabías en un puño hasta que necesité las dos manos que ya ahora no me alcanzan [Paraguay: Gayosa, Milia, Ronda en las olas, 1990]

(21) a. Esa vida y esa masacre no duraron horas, ni días, sino años, se necesitó de años para matar tantos millones de seres [Paraguay: Gertopan, S., Barrio palestina 1998] $^{\circ}$ 
b. En la novela moderna, el genio de Kafka necesitó de Orson Welles para que fuera puesto en una pantalla El proceso [Argentina: Diario La Nación, 1992]

c. Según informes preliminares, la gestión de Chito Valle no necesitó más de 2 días para iniciar una serie de irregularidades durante su nombramiento transitorio como Alcalde de la ciudad de La Paz [Bolivia: ERBOL, 01/27/69]

En suma, el parámetro del aspecto imperfectivo tampoco permite explicar la alternancia entre el régimen directo y prepositivo en nuestros tres verbos analizados.

\subsection{Apoyo cuantitativo}

Como ya mencionamos, en una primera etapa del análisis buscamos establecer los factores que condicionaran la alternancia dentro de los elementos que constituyen la construcción, para lo cual se realizó un análisis cuantitativo de los factores que, según la bibliografía, atraen la presencia de construcciones antipasivas. Como mostraremos a continuación, la revisión cuantitativa no nos permite explicar la alternancia entre régimen directo y preposicional de manera contundente.

Como hemos visto, uno de los parámetros para la presencia de la antipasiva es el grado de completud del evento. Como observamos en los ejemplos de (8), las construcciones negativas indican que se trata de un evento incompleto, lo que, de acuerdo con el criterio mencionado, atraería una construcción antipasiva. Por tanto, el factor relativo al evento completo e incompleto se analizó por medio del contraste entre oraciones afirmativas o positivas y construcciones negativas. Los datos mostraron que no hay un contraste entre formas afirmativas y negativas, como se ilustra en la Tabla 2.

Tabla 2. Evento positivo vs. negado

\begin{tabular}{|l|c|c|c|c|}
\hline & \multicolumn{2}{|c|}{ EVENTO POSITIVO } & \multicolumn{2}{c|}{ EVENTO NEGADO } \\
\hline & CD & CP & CD & CP \\
\hline Cuidar & $132 / 181$ & $49 / 181$ & $2 / 2$ & $0 / 2$ \\
& $72.92 \%$ & $27.07 \%$ & $100 \%$ & --- \\
\hline Necesitar & $292 / 363$ & $71 / 363$ & $42 / 56$ & $14 / 56$ \\
& $80.44 \%$ & $19.55 \%$ & $75 \%$ & $25 \%$ \\
\hline Disfrutar & $46 / 332$ & $286 / 332$ & $4 / 13$ & $9 / 13$ \\
& $13.85 \%$ & $86.14 \%$ & $30.76 \%$ & $69.23 \%$ \\
\hline
\end{tabular}


Las construcciones negativas fueron escasas, cuidar y necesitar muestran que tanto los casos afirmativos como los negativos tienden a un régimen directo. De igual manera, el verbo disfrutar no muestra restricciones respecto a este factor para seleccionar preferentemente el régimen preposicional.

El segundo factor que atañe a las construcciones antipasivas es la falta de individuación del objeto. Si nuestros verbos pudiesen explicarse en términos de una diátesis antipasiva, las construcciones con régimen directo deberían presentar en su mayoría un alto grado de individuación, al contrario de las construcciones con régimen prepositivo. Por lo anterior, realizamos un análisis cuantitativo del grado de determinación del referente del complemento, el cual se muestra en la Tabla 3:

Tabla 3. Determinación de los $C D$ vs. $C P$

\begin{tabular}{|l|c|c|c|c|}
\hline & \multicolumn{2}{|c|}{ DETERMINADO } & \multicolumn{2}{c|}{ INDETERMINADO } \\
\hline & $\mathrm{CD}$ & $\mathrm{CP}$ & $\mathrm{CD}$ & $\mathrm{CP}$ \\
\hline Cuidar & $119 / 156=$ & $47 / 156=$ & $15 / 17=$ & $2 / 17=$ \\
& $76 \%$ & $30 \%$ & $88 \%$ & $11 \%$ \\
\hline \multirow{2}{*}{ Necesitar } & $82 / 119=$ & $37 / 119=$ & $252 / 300=$ & $48 / 300=$ \\
& $68.9 \%$ & $31 \%$ & $84 \%$ & $16 \%$ \\
\hline \multirow{2}{*}{ Disfrutar } & $44 / 203=$ & $159 / 203=$ & $6 / 142=$ & $136 / 142=$ \\
& $21.6 \%$ & $78.3 \%$ & $4.2 \%$ & $95.7 \%$ \\
\hline
\end{tabular}

Los resultados arrojados por el corpus muestran que la alternancia no parece estar condicionada por este factor de la determinación del segundo participante; tal como lo muestra la tabla. Para los verbos cuidar y necesitar, el régimen directo vale tanto si el complemento se trata de una entidad determinada como no determinada. Solo el verbo disfrutar tiende, al contrario, a la elección del régimen preposicional, pero del mismo modo que los otros verbos, el factor de determinación no parece ser restrictivo para la configuración sintáctica del complemento.

Para analizar el factor de individuación también recurrimos a los criterios de Hopper y Thompson (1980), la cual considera que la individuación es un compuesto de las siguientes propiedades binarias del nombre y en la cual los referentes nominales con las propiedades de la izquierda se encuentran más individuados que los de la derecha:

Propio / común

Humano, animado / inanimado

Definido / no definido 
Referencial/ no referencial

Singular/ plural

Numerable / masa

Estos factores analizados no mostraron información contundente para explicar el fenómeno que nos atañe. Por ejemplo, aspectos como la indeterminación o el menor grado de individualización (plural) no parecen mostrarse como condiciones propias de un $\mathrm{P}$ oblicuo, ya que las mismas tendencias de frecuencia relativa de usos se encontraron en construcciones con objeto directo. ${ }^{4}$

Para ejemplificar lo anterior, tenemos el contraste entre el colectivo, el singular y el plural. Como se puede observar en la Tabla 4, este factor de individuación tampoco constituyó un aspecto relevante en la alternancia régimen directo - preposicional; los verbos cuidar y necesitar tienden al régimen directo con cualquier tipo de entidad; mientras que disfrutar prefiere el régimen preposicional sin atender si el complemento es un colectivo, singular o plural.

Tabla 4. Colectivo/ singular / plural

\begin{tabular}{|l|c|c|c|c|c|c|}
\hline & \multicolumn{2}{|c|}{ COLECTIVO } & \multicolumn{2}{c|}{ SINGULAR } & \multicolumn{2}{c|}{ PLURAL } \\
\hline & CD & CP & CD & CP & CD & CP \\
\hline Cuidar & $1 / 2=$ & $1 / 2=$ & $77 / 106=$ & $29 / 106=$ & $56 / 72=$ & $19 / 72=$ \\
& $50 \%$ & $50 \%$ & $72.6 \%$ & $27.3 \%$ & $77.7 \%$ & $26.3 \%$ \\
\hline Necesitar & --- & $3 / 3=$ & $249293=$ & $44 / 293=$ & $85 / 123=$ & $38 / 123=$ \\
& & $100 \%$ & $84.9 \%$ & $15 \%$ & $69.1 \%$ & $30.89 \%$ \\
\hline Disfrutar & $1 / 4=$ & $3 / 4=$ & $38 / 267=$ & $229 / 267$ & $11 / 74=$ & $63 / 74=$ \\
& $25 \%$ & $75 \%$ & $14.2 \%$ & $=85.7 \%$ & $14.86 \%$ & $85.1 \%$ \\
\hline
\end{tabular}

En la tabla se puede observar, una vez más, que cuidar y necesitar, en cuanto a la variable singular y plural, tienden al régimen directo: cuidar con $72.6 \%$ de casos con singular y $77.7 \%$ de los casos con plural, mientras que necesitar con $84.9 \%$ de casos con singular y $69.1 \%$ con plural. El verbo disfrutar, que sigue la tendencia contraria al preferir el régimen prepositivo, presentó $85.7 \%$ de los casos de régimen con singular y $85.1 \%$ con casos en plural. El corpus arrojó muy pocos casos con referentes que se identifican con entidades colectivas: cuidar mostró números equivalentes al mostrar solo dos datos (50\% en cada caso); necesitar, únicamente tres casos que seleccionaron preposición (100\%) y disfrutar que siguió claramente la tendencia hacia el régimen preposicional $(75 \%)$.

\footnotetext{
${ }^{4}$ Por cuestiones de espacio, solo presentaremos algunos de los factores analizados.
} 
Otro factor que nos permite ejemplificar el grado de individuación es el contraste entre entidades humanas y no humanas. Como hemos señalado, las entidades humanas tienden a ser más individuadas que las no humanas, por lo que, atendiendo a los parámetros de la antipasiva, se esperaría que, tratándose de referentes humanos, las construcciones prefirieran el régimen directo. Sin embargo, los datos arrojaron que este factor tampoco incide en la elección de un tipo determinado tipo de régimen, como se ilustra en la Tabla 5.

Tabla 5. Animacidad de los $C D$ vs. $C P$

\begin{tabular}{|l|c|c|c|c|}
\hline & \multicolumn{2}{|c|}{ Humano } & \multicolumn{2}{c|}{ No Humano } \\
\hline & CD & CP & CD & CP \\
\hline Cuidar & $53 / 77=$ & $24 / 77=$ & $81 / 106=$ & $25 / 106=$ \\
& $68.8 \%$ & $31.1 \%$ & $76.4 \%$ & $26.4 \%$ \\
\hline \multirow{2}{*}{ Necesitar } & $53 / 73=$ & $20 / 73=$ & $281 / 346=$ & $65 / 346=$ \\
& $72.6 \%$ & $27.3 \%$ & $81.2 \%$ & $18.7 \%$ \\
\hline Disfrutar & $1 / 9=$ & $8 / 9=$ & $49 / 336=$ & $287 / 336=$ \\
& $11.1 \%$ & $88.8 \%$ & $14.5 \%$ & $85.4 \%$ \\
\hline
\end{tabular}

El tercer factor que condiciona la diátesis antipasiva es el aspecto imperfectivo. Esta variable del aspecto se analizó a través de la revisión entre los tiempos verbales de mayor frecuencia en el corpus, a saber: presente, copretérito y pretérito, los dos primeros aspectualmente imperfectivos y el último, perfectivo. Los datos mostraron poca diferencia a lo presentado en los factores anteriores, tal como se observa en la Tabla 6.

Tabla 6. Aspecto imperfectivo: presente, copréterito $\leftrightarrow \rightarrow$ pretérito

\begin{tabular}{|l|c|c|c|c|c|c|}
\hline & \multicolumn{2}{|c|}{ PRESENTE } & \multicolumn{2}{c|}{ COPRETÉRITO } & \multicolumn{2}{c|}{ PRETÉRITO } \\
\hline & CD & CP & CD & CP & CD & CP \\
\hline Cuidar & $34 / 52=$ & $18 / 52=$ & $37 / 53=$ & $16 / 53=$ & $11 / 15=$ & $4 / 15=$ \\
& 65.38 & 34.61 & 69.81 & 30.18 & 73.33 & 26.66 \\
\hline \multirow{2}{*}{ Necesitar } & $147 / 190=$ & $43 / 190=$ & $103 / 119=$ & $16 / 119=$ & $18 / 26=$ & $8 / 26=$ \\
& 77.36 & 22.63 & 86.55 & 13.44 & 69.23 & 30.76 \\
\hline Disfrutar & $13 / 92=$ & $79 / 92=$ & $14 / 89=$ & $75 / 89=$ & $10 / 91=$ & $81 / 91=$ \\
& 14.13 & 85.86 & 15.73 & 84.26 & 10.98 & 89.01 \\
\hline
\end{tabular}

De poderse explicar la alternancia como un fenómeno de diátesis antipasiva, los tiempos imperfectivos (el presente y copretérito) atraerían, desde el punto de vista porcentual, al régimen prepositivo, mientras que el pretérito al régimen directo. Sin embargo, como se puede observar en los porcentajes de la tabla, no se presenta la correlación entre imperfectividad y régimen preposicional, así como entre perfectividad y régimen directo. 
A manera de síntesis de esta sección, podemos decir que el análisis cuantitativo de la completud del evento, el nivel de individuación y el aspecto no muestran datos contundentes para explicar la alternancia entre régimen directo y prepositivo. Así, los factores que atraen la diátesis antipasiva no se presentan de manera significativa estadísticamente en las construcciones con régimen prepositivo.

\subsection{Pares mínimos}

Otro argumento a favor de que las construcciones que constituyen nuestro objeto de estudio no pueden ser explicadas en términos de la diátesis antipasiva es el contraste entre pares mínimos. Un mismo contexto se puede codificar tanto con régimen directo como preposicional. Nos referimos a un par mínimo que presenta el mismo verbo y el complemento presenta el mismo núcleo de la frase nominal (por ejemplo: disfruta el / un apoyo vs. disfruta del / de un gran apoyo).

El primer par mínimo que ofrecemos como ejemplo es disfrutar + el sustantivo vida, el cual nos permitirá contrastar el rasgo de completud del evento. Un evento completo, de acuerdo con los parámetros de la antipasiva, debería atraer un régimen directo. Sin embargo, tenemos casos en que un mismo evento completo se codifica tanto con CD como con CP, como en (22), donde el adverbio mucho indica la completud del evento.

(22) a. Este mundo después de todo es muy divertido y al menos o disfruto mucho esta vida, mira que me río [Cuba, jovencuba.com]

b. Esta mujer hizo grandes cosas: vivió y disfrutó muchode la vida [Chile, Amó historias verdaderas amores que perduran]

De manera análoga, encontramos pares mínimos en donde ambas oraciones se refieren a eventos incompletos. En (23), por ejemplo, las oraciones negativas nos indican un evento incompleto y, pese a ello, en un caso se selecciona el régimen directo y en otro el indirecto.

(23) a. Así, se ha empezado a comprar objetos como teléfonos celulares diminutos, gruesos relojes de titanio o pantalones Levis genuinos. Pero no disfruta del todo su vida [Perú, Pablo Garssia, "Polilla", 2000]

b. La persona tímida, al tener esa distorsión de la realidad, no disfruta de la vida ya que la ve como un peligro constante [España, Francisco Javier Salazar, “Cómo vencer la timidez...”] 
Con respecto al parámetro de individuación, encontramos pares mínimos con régimen directo y prepositivo en donde ambas oraciones son altamente individuadas y, pese a ello, pueden codificar ambos tipos de régimen, cuando lo esperable sería el régimen directo. Veamos el par mínimo de (24), en donde el determinante posesivo otorga un

carácter más definido a los referentes, en tanto que los hace identificables para el oyente.

(24) a. Qué curiosa la similitud [...] que presenta este ámbito del ser el de la niñez, con el ámbito del manicomio (unos siglos atrás y aún hoy), donde el loco era considerado un niño, un pupilo bajo la tutela del Estado o el ojo guardián del médico que cuidaba paternalmente a sus hijos, los insanos, como de su familia [Paraguay, López, Nila:Señales: una intrahistoria, 1954]

b. Volviéndose hacia el lloroso Tane, que la había seguido, le dijo que mientras él cuidara de sus hijos en la tierra, ella siempre los arrastraría a reunirse con ella en el infierno [Enc: Leyendas maories]

Asimismo, encontramos pares mínimos en donde ambas oraciones son poco individuadas, lo que tampoco incide en la elección de uno u otro régimen. Por ejemplo, en (25) el referente del complemento de ambas oraciones (niños) es poco individuado, pues en (25a) es indefinido y en (25b) es genérico. Tal falta de individuación supondría la presencia de un CP; no obstante, ambas oraciones codifican diferente tipo de régimen.

a. Entonces, gente, por ejemplo una chica que cuidaba niños en Los Ángeles, Puritanismo y vale [España oral: CCON031B]

b. Son las mujeres quienes sostienen nuestra tierra; las que plantan y cosechan; las que tejen, las que cuidan de los niños y de los animales [Paraguay: Lebron, Maybell, Pancha, 2000]

En cuanto al aspecto verbal, también encontramos pares mínimos de régimen directo versus preposicional que apoyan nuestro planteamiento. En (26), encontramos oraciones en presente habitual, tiempo por excelencia imperfectivo, lo que debería atraer el régimen prepositivo; no obstante, (26a) presenta régimen directo: 
(26) a. la mayor parte de los proyectos de campo necesitan el apoyo de diferentes profesionales, incluidos los asesores estadísticos, biólogos, sociólogos y estudiantes colaboradores [Enc: Musicología]

b. Esta investigación necesita del apoyo de instituciones sociales y políticas, constructores de instrumentos, copia e impresión de música, y la interpretación de esa música para obtener solamente algunos ejemplos. [Enc: Musicología]

Asimismo, encontramos pares mínimos en los que ambas oraciones presentan un aspecto perfectivo, como en (27), donde ambas oraciones se encuentran en pretérito de indicativo:

(27) a. La película, una coproducción de Reino Unido, Alemania y Canadá que necesitó el apoyo de hasta 14 entidades diferentes para reunir un ajustado presupuesto de 15 millones [joseacuenca.espacioblog.com ]

b. Los papas necesitaron del apoyo imperial para sofocar varios levantamientos en Roma [Enc: Alemania]

Como vemos, estos pares mínimos ponen en duda el hecho de que el fenómeno de la alternancia entre régimen directo y preposicional se pueda explicar en términos de una diátesis antipasiva, pues un par de contextos similares en términos referenciales y lingüísticos admiten ambos tipos de régimen.

\section{Nota final: otros factores a considerar}

Como se ha observado, el análisis a la luz de los parámetros de la antipasiva no fue determinante para explicar la alternancia entre régimen directo y preposicional que muestran las construcciones con los verbos estudiados. Una posible explicación de la alternancia descansa en las diferencias dialectales. Recurrimos a una cala de datos para comparar los dialectos de México y España. Para ello, para cada verbo y dialecto, tomamos una muestra aleatoria de 200 ejemplos del corpus de Mark Davies. Los resultados de dicha muestra se muestran a continuación: 
Tabla 7: Distribución de la alternancia CD vs. CP en México y España

\begin{tabular}{|l|c|c|c|c|}
\hline & \multicolumn{4}{|c|}{ País } \\
\hline Verbo & \multicolumn{2}{|c|}{ México } & España \\
\hline & CD & CP & CD & CP \\
\hline Disfrutar & $81 / 200=$ & $119 / 200$ & $30 / 200=$ & $170 / 200=$ \\
& $40.5 \%$ & $=59.5 \%$ & $15 \%$ & $85 \%$ \\
\hline Necesitar & $172 / 200=$ & $28 / 200=$ & $183 / 200=$ & $17 / 200=8$ \\
& $86 \%$ & $14 \%$ & $91.5 \%$ & $.5 \%$ \\
\hline Cuidar & $175 / 200=$ & $25 / 200=$ & $153 / 200=$ & $47 / 200=$ \\
& $87.5 \%$ & $12.5 \%$ & $76.5 \%$ & $23.5 \%$ \\
\hline
\end{tabular}

Como se puede observar, el dialecto peninsular muestra una preferencia por el régimen preposicional en los verbos disfrutar, contrario a lo que ocurre con el verbo necesitar. Nótese que las diferencias dialectales más significativas se encuentran en el verbo disfrutar, en donde la brecha de porcentajes es significativa. Sin embargo, en los verbos necesitar y cuidar la variación no fue tan contundente. No obstante, se requiere un análisis dialectal más exhaustivo para determinar si el factor diatópico determina la alternancia. Para ello también tendrían que someterse a comparación diversos dialectos del español, como el argentino, por ejemplo.

Asimismo, queda pendiente para trabajos posteriores una revisión del género discursivo, pues posiblemente podría ser otra alternativa para analizar el fenómeno que nos ocupa, ya que se ha visto que el comportamiento de ciertos fenómenos sintácticos se puede explicar en términos discursivos, como es el caso de las construccionesde sujeto y objeto experimentantes que analiza Vázquez y Miglio (2016).

\section{Conclusiones}

En este trabajo analizamos tres verbos que presentan alternancia entre el régimen directo y preposicional, necesitar, cuidar y disfrutar, con la intención de establecer los factores que condicionan dicha alternancia. Los verbos analizados son semánticamente heterogéneos, pero, sintácticamente, comparten la propiedad de alternar entre el régimen directo y el preposicional.

En un primer momento, planteamos la posibilidad de explicar la alternancia como un fenómeno de diátesis en tanto que un complemento directo de carácter central se vuelve un argumento oblicuo con la función de complemento prepositivo, lo cual en un nivel semántico-pragmático sería el reflejo de un distanciamiento entre el sujeto y el efecto de la predicación sobre el complemento degradado. Por lo anterior, con el fin de comprobar o no 
la hipótesis planteada, llevamos a cabo un análisis a la luz de los parámetros que caracterizan la función de VOz antipasiva: la no completud del evento, falta de individuación y la imperfectividad aspectual. Estos factores no fueron contundentes para explicar el fenómeno que nos atañe, pues la elección de una u otra forma de régimen se mostró indiferente a cada uno de los parámetros analizados.

En lo que respecta a la completud del evento, lo esperable es que las construcciones afirmativas que expresan la realización de la predicación seleccionen un régimen directo, mientras que la presencia de CP sería esperable cuando no se lleva a cabo el evento o estado de cosas que predica el verbo. Sin embargo, se encontraron datos que expresan eventos alcanzados cuyo complemento se formaliza como $\mathrm{CP}$, así como eventos no alcanzados del todo o parcialmente alcanzados cuya predicación se realiza mediante el régimen directo.

Las construcciones antipasivas, además, suponen que los complementos poseen un bajo grado de individuación, por lo que es esperable que aparezcan introducidos por una marca morfológica o prepositiva. Las estructuras activas, por su parte, preferirían un régimen directo con objetos con un alto grado de individuación. Los datos demostraron, no obstante, que el parámetro de individuación tampoco se cumple sistemáticamente, pues fue posible encontrar datos que presentan objetos altamente individuados introducidos con la preposición de, así como objetos con baja individuación con régimen directo.

El último parámetro que se revisó se relaciona con la dimensión aspectual. Las construcciones antipasivas suelen presentarse en construcciones imperfectivas, mientras que los verbos en tiempos que nos remiten a modos aspectuales perfectivos tienden a elegir el régimen directo. Los datos, una vez más, evidenciaron el incumplimiento del parámetro, ya que se encontraron eventos puntuales con régimen prepositivo.

Los parámetros de la antipasiva también se revisaron desde el punto de vista cuantitativo. Del total de datos recolectados, se pudo observar que los verbos necesitar y cuidar mostraron mayor preferencia por la rección directa, mientras que disfrutar tiende al régimen preposicional. Estas preferencias se mantuvieron constantes en la revisión estadística de los tres factores que condicionan la antipasiva (la no completud del evento, falta de individuación y la imperfectividad aspectual).

Estos tres factores se analizaron a partir de variables específicas. El factor relativo al evento completo e incompleto se analizó a través del contraste entre oraciones afirmativas o positivas y construcciones negativas, la falta de individuación del objeto a partir de la escala de individuación de Hopper y Thompson (1980) y la imperfectividad aspectual mediante el 
contraste de tiempos verbales. Ninguna de estas variables mostró datos contundentes para explicar la alternancia entre régimen directo y prepositivo.

Otro argumento aducido para poner en evidencia que la diátesis antipasiva no permite explicar la alternancia fue el contraste entre pares mínimos. Se contrastó el rasgo de completud del evento con ejemplos en que se habla de un mismo evento completo, así como con pares de oraciones que se refieren a eventos incompletos y se obtuvo que en ambos casos es posible codificar tanto con CD como con CP. De manera análoga, en lo que respecta al parámetro de individuación se encontraron pares mínimos con régimen directo y prepositivo en donde ambas oraciones son altamente individuadas y, pese a ello, pueden codificar ambos tipos de régimen. También se encontraron pares mínimos en donde ambas oraciones eran poco individuales y esto no incidió en la elección de un régimen u otro. En cuanto al aspecto verbal, la elección de un régimen u otro fue indiferente al hecho de que los verbos fueran perfectivos o no.

En suma, el análisis a través de los parámetros de la antipasiva no fue determinante para explicar la alternancia que muestran las construcciones con los verbos estudiados entre régimen directo y preposicional. Por ello, es menester buscar la explicación del fenómeno a través de otras alternativas, como las diferencias dialectales, que hemos esbozado muy brevemente, o bien, diferencias discursivas.

Nos cuestionamos entonces, si el fenómeno encuentra explicación en el propio sistema, es decir, si hay factores estrictamente lingüísticos que determinan el fenómeno. De ser así, cabría preguntarse por qué habiendo pares mínimos con contextos referenciales y sintácticos tan similares se presenta la alternancia. También existe la posibilidad de que el fenómeno encuentre explicación en una variación libre. Para determinar lo anterior, se necesitan estudios posteriores más exhaustivos que agoten un inventario de factores lingüísticos más amplio. De igual manera, cabe la posibilidad de que el fenómeno obedezca a factores extralingüísticos, como el dialecto, el registro y el género. Así pues, este objeto de estudio es susceptible de múltiples estudios posteriores. La aportación de este trabajo es el haber explorado una de las vías de análisis del fenómeno.

\section{Referencias}

Alarcos Llorach, Emilio. 1978. Estudios de gramática funcional del español. Madrid: Gredos. Bogard, Sergio. 1999. Construcciones antipasivas en español. Nueva Revista de Filología Hispánica 2. 305-327. 
Cano Aguilar, Rafael. 1999. Los complementos de régimen verbal. En Bosque, Ignacio \& Demonte, Violeta(dirs.), Gramática descriptiva de la lengua española, vol. 2. 1807-1854. Madrid: Espasa Calpe.

Cifuentes Honrubia, José Luis. 2010. Clases semánticas y construcciones sintácticas: alternancias locales en español. Lugo: Axac.

Cooreman, Ann. 1994. A Functional Typology of Antpassives.En B. Fox \& P. J. Hopper (eds.), Voice.Form and Function. Amsterdam/Philadelphia: John Benjamins.

García Miguel, José María. 1995. Las relaciones gramaticales entre sujeto y participantes. Santiago de Compostela: Universidad de Santiago de Compostela.

Givón, Thomas. 1984. Syntax. A Functional-Typological Introduction,Vol. I. Amsterdam/Philadelphia: John Benjamins.

Hopper, Paul J. \& Thompson, Sandra Annear.1980. Transitivity in grammar and discourse. Language 56(2). 251-299. DOI: 10.2307/413757

Jiménez Norberto, Laura. 2009. El estatus argumental del complemento de régimen prepositivo en español. México: Universidad Autónoma Metropolitana (tesis de maestría).

Maldonado Soto, Ricardo. 2004. ¿Voz media o voz antipasiva? Una mirada cognoscitiva. En Serra, Enrique \& Wotjak, Gerd (eds.), Cognición y percepción linguïsticas, 117-135, Valencia/Leipzig: Univesitat de Valencia/Universität Leipzig.

Moreno Cabrera, Juan Carlos. 1984. La diátesis anticausativa. Ensayo de sintaxis general, Revista Española de Lingüistica 14(1). 21-44.

Rojas Nieto, Cecilia. 1988. Verbos locativos en español. Aproximación sintáctico-semántica. México: UNAM.

Vázquez Rozas, Victoria \&Miglio, Viola G. 2016. Constructions with subject vs. object experiencers in Spanish and Italian. En Yoon, Jiyoung. \&Gries, Stefan (eds.), Corpusbased Approaches to Construction Grammar, vol. 19.65-102. Amsterdam: John Benjamins

\section{CORPUS}

Davies, Mark. Corpus del español: 100 millones de palabras, siglo XIII - siglo XX. En construcción desde el año 2002, http://www.corpusdelespanol.org [consultado en julio de $2015 \mathrm{y}$ diciembre de 2016]

Universidad de Vigo. Base de datos de Verbos, Alternancias de Diátesis y Esquemas SintácticoSemánticos del Español, http://adesse.uvigo.es/ [consultado en julio de 2015]

\section{Colaboración}

Agradecemos las observaciones y sugerencias de la Dra. Chantal Melis, investigadora del Instituto de Investigaciones Filológicas de la UNAM. Por supuesto, todas las fallas de este trabajo son atribuibles a las autoras. 
Blanca Elena Sanz Martin es profesora e investigadora del Depto. de Letras de la Universidad Autónoma de Aguascalientes (México). Sus principales líneas de investigación giran en torno a la sintaxis y la semántica en lengua española. Ha sido autora de diversos artículos y capítulos de libros publicados en México, Latinoamérica y Europa, así como del libro El proceso de auxiliarización del verbo tener. De la posesión al aspecto. Actualmente es responsable del proyecto de investigación "Contenido léxico, estructura argumental y realización sintáctica del verbo ver".

Cristina Eslava Heredia es profesora del Departamento de Letras de la Universidad Autónoma de Aguascalientes, México. Su ámbito de investigación es la sintaxis histórica y es autora del libro Los verbos de movimiento en el español alfonsí (Universidad autónoma de Aguascalientes, 2013). Ha publicado trabajos sobre los verbos de movimiento y la alternancia preposicional en la historia del español peninsular y de México. Actualmente, es responsable del proyecto "Caracterización sintáctico-semántica de los verbos de pensamiento en la historia del español”. 